Proyecciones Journal of Mathematics

Vol. 36, No 3, pp. 485-498, September 2017.

Universidad Católica del Norte

Antofagasta - Chile

\title{
A generalization of variant of Wilson's type Hilbert space valued functional equations
}

\author{
Hajira Dimou \\ University of Ibn Tofail, Morocco \\ and \\ Samir Kabbaj \\ University of Ibn Tofail, Morocco \\ Received : December 2016. Accepted : May 2017
}

\begin{abstract}
In the present paper we characterize, in terms of characters, multiplicative functions, the continuous solutions of some functional equations for mappings defined on a monoid and taking their values in a complex Hilbert space with the Hadamard product. In addition, we investigate a superstability result for these equations.
\end{abstract}

Keywords : D'Alembert's functional equation, Hilbert space, Hadamard product, superstability.

2010 Mathematics Subject Classifications : Primary, 39B72, 39B82; Secondary $46 E 40$. 


\section{Introduction}

Let $M$ be a monoid i.e., is a semigroup with an identify element that we denote by e and $\sigma, \tau: M \rightarrow M$ are two involutive automorphisms. That is $\sigma(x y)=\sigma(x) \sigma(y), \tau(x y)=\tau(x) \tau(y)$ and $\sigma(\sigma(x))=x, \tau(\tau(x))=x$ for all $x, y \in M$. By a variant of Wilson's functional equation on $M$ we mean the functional equation

$$
f(x \sigma(y))+f(\tau(y) x)=2 f(x) g(y), \quad x, y \in M,
$$

where $f, g: M \rightarrow \mathbf{C}$ are the unknown functions. A special case of Wilson's functional equation is d'Alembert's functional equation:

$$
f(x \sigma(y))+f(\tau(y) x)=2 f(x) f(y), \quad x, y \in M,
$$

The solutions of equation (1.2) are known [2]. Further contextual and historical discussion on the functional equation (1.1) and (1.2) can be found, e.g., in [6.2].

The present paper studies an extension to a situation where the unknown functions $\mathrm{f}$, g map a possibly non-abelian group or monoid into a complex Hilbert space $H$ with the Hadamard product. Our considerations refer mainly to results by Rezaei [4], Zeglami [11]. It has been proved [3] that the functional equation (1.2) with $\sigma=i d$ is superstable in the class of functions $f: G \rightarrow \mathbf{C}$, if every such function satisfies the inequality

$$
|f(x y)+f(\tau(y) x)-2 f(x) f(y)| \leq \epsilon \text { for all } x, y \in G,
$$

where $\epsilon$ is a fixed positive real number. Then either $f$ is a bounded function or

$$
f(x y)+f(\tau(y) x)=2 f(x) f(y), \quad x, y \in G .
$$

Let $H$ be a separable Hilbert space with a orthonormal basis $\left\{e_{n}, n \in\right.$ $\mathbf{N}$ \}. For two vectors $x, y \in H$, the Hadamard product, also known as the entrywise product on the Hilbert space $H$ is defined by

$$
x * y=\sum_{n=0}^{\infty}\left\langle x, e_{n}\right\rangle\left\langle y, e_{n}\right\rangle e_{n}, \quad x, y \in H .
$$

The Cauchy-Schwarz inequality together with the Parseval identity ensure that the Hadamard multiplication is well defined. In fact,

$$
\|x * y\| \leq\left(\sum_{n=0}^{\infty}\left|\left\langle x, e_{n}\right\rangle\right|^{2}\right)^{\frac{1}{2}}\left(\sum_{n=0}^{\infty}\left|\left\langle y, e_{n}\right\rangle\right|^{2}\right)^{\frac{1}{2}}=\|x\|\|y\| .
$$


The purpose of this work is first to give a characterization, in terms of multiplicative functions, the solutions of the Hilbert space valued functional equation by Hadamard product:

$$
f(x \sigma(y))+f(\tau(y) x)=2 g(x) * f(y), \quad x, y \in M .
$$

When $\mathrm{f}$ we determine the solutions of the functional equation

$$
f(x \sigma(y))+f(\tau(y) x)=2 f(x) * g(y), \quad x, y \in M,
$$

where $f, g: M \rightarrow H$ are the unknown functions. Second, we determine a characterization of the following d'Alembert-Hilbert-valued functional equation:

$$
f(x \sigma(y))+f(\tau(y) x)=2 f(x) * f(y), \quad x, y \in M .
$$

Throughout the paper, $\mathbf{N}, \mathbf{R}$ and $\mathbf{C}$ stand for the sets of positive integers, real numbers and complex numbers, respectively. We let $G$ denote a group and $S$ denote a semigroup i.e., a set with an associative composition rule.

A function $A: M \rightarrow \mathbf{C}$ is called additive, if it satisfies $A(x y)=$ $A(x)+A(y)$ for all $x, y \in M$.

A multiplicative function on $M$ is a map $\chi: M \rightarrow \mathbf{C}$ such that $\chi(x y)=\chi(x) \chi(y)$ for all $x, y \in M$.

A monoid $M$ is generated by its squares if for every $x \in I_{\chi}, x=$ $x_{1}^{2} x_{2}^{2} \cdots x_{n}^{2}$ for some $x_{1}, x_{2}, \cdots, x_{n} \in M$.

A character on a group $G$ is a homomorphism from $G$ into the multiplicative of non-zero complex numbers. While a non-zero multiplicative function on a group can never take the value 0 , it is possible for a multiplicative function on a monoid $M$ to take the value 0 on a proper, non-empty subset of $M$. If $\chi: M \rightarrow \mathbf{C}$ is multiplicative and $\chi \neq 0$, then

$$
I_{\chi}=\{x \in M / \chi(x)=0\}
$$

is either empty or a proper subset of $M$. The fact that $\chi$ is multiplicative establishes that $I_{\chi}$ is a two-sided ideal in $M$ if not empty (for us an ideal is never the empty set). It follows also that $M \backslash I_{\chi}$ is a subsemigroup of $M$.

Let $C(M)$ denote the algebra of continuous functions from $M$ into $\mathbf{C}$. 


\section{Solutions of (1.5) and (1.6)}

In this section, we solve the functional equation (1.5) by expressing its solutions in terms of multiplicative functions.

Theorem 2.1. Let $M$ be a monoid, let $\sigma, \tau: M \rightarrow M$ be involutive automorphisms. Assume that the functions $f, g: M \rightarrow H$ satisfy (1.5). Then, there exists a positive integer $N$ such that

$$
f(x)=\sum_{n=1}^{N}\left\langle f(x), e_{n}\right\rangle e_{n} \text { and } x \rightarrow\left\langle g(x), e_{N+k}\right\rangle \text { is arbitrary }
$$

for all $x \in M$ and $k>0$. Furthermore, for every $k \in\{1,2, \ldots, N\}$, we have the following possibilities:

$\left\{\begin{array}{l}\left\langle g(x), e_{k}\right\rangle=\frac{\chi_{k}(x)+\chi_{k} \circ \sigma \circ \tau(x)}{2} \\ \left\langle f(x), e_{k}\right\rangle=\frac{\alpha_{k}\left(\chi_{k}(x)+\chi_{k} \circ \sigma \circ \tau(x)\right)}{2}\end{array} ;\left\{\begin{array}{l}\left\langle g(x), e_{k}\right\rangle \text { is an arbitrary function, } \\ \left\langle f(x), e_{k}\right\rangle=0\end{array}\right.\right.$

for all $x \in M$, where $\chi_{k}$ is a non-zero multiplicative function of $M$ such that $\chi_{k} \circ \sigma \circ \tau=\chi_{k} \circ \tau \circ \sigma$ and $\alpha_{k} \in \mathbf{C} \backslash\{0\}$. If $M$ is a topological monoid and $f \in C(M)$, then $\chi_{k}, \chi_{k} \circ \sigma \circ \tau \in C(M)$.

Proof. For every integer $k \geq 0$, consider the functions $f_{k}, g_{k}: M \rightarrow \mathbf{C}$ defined by

$$
f_{k}(x)=\left\langle f(x), e_{k}\right\rangle \text { and } g_{k}(x)=\left\langle g(x), e_{k}\right\rangle \text { for all } x \in M \text {. }
$$

Since $(f, g)$ satisfies (1.5), for all $x, y \in M$, we have

$$
\begin{aligned}
\sum_{k=0}^{+\infty}\left\{\left\langle f(x \sigma(y)), e_{k}\right\rangle+\left\langle f(\tau(y) x), e_{k}\right\rangle\right\} e_{k} & =\sum_{k=0}^{+\infty}\left\langle\{f(x \sigma(y))+f(\tau(y) x)\}, e_{k}\right\rangle e_{k} \\
& =f(x \sigma(y))+f(\tau(y) x) \\
& =2 g(x) * f(y) \\
& =2 \sum_{k=0}^{+\infty}\left\langle g(x), e_{k}\right\rangle\langle f\rangle,
\end{aligned}
$$

This yields for all $k \in \mathbf{N}$,

$$
f_{k}(x \sigma(y))+f_{k}(\tau(y) x)=2 g_{k}(x) f_{k}(y) \text { for all } x, y \in M \text {. }
$$


If we put $y=e$ in $(2.1)$, we find that $f_{k}(x)=f_{k}(e) g_{k}(x)$. So, if we take $\alpha_{k}=f_{k}(e)$, equation (2.1) can be written as follows:

$$
\alpha_{k} g_{k}(x \sigma(y))+\alpha_{k} g_{k}(\tau(y) x)=2 \alpha_{k} g_{k}(x) g_{k}(y) \text { for all } x, y \in M \text {. }
$$

Then, either $\alpha_{k}=0$ or $g_{k}$ is a solution of equation (1.6). In view of [2, Theorem 3.2], one of the following statements holds:

(a) We have that

$$
f_{k}=0 \text { and } g_{k} \text { is an arbitrary function. }
$$

(b) There exists a multiplicative function $\chi_{k}$ such that

$g_{k}(x)=\frac{\chi_{k}(x)+\chi_{k} \circ \sigma \circ \tau(x)}{2}$ and $f_{k}(x)=\frac{\alpha_{k}\left(\chi_{k}(x)+\chi_{k} \circ \sigma \circ \tau(x)\right)}{2}$ for $x \in M$.

If $H$ is infinite-dimensional, then

$$
\left\langle g(x), e_{k}\right\rangle=g_{k}(x) \rightarrow 0 \text { as } k \rightarrow+\infty
$$

for every $x \in M$. Since $g_{k}(e)=1$, statement (b) is not possible for infinitely many positive integers $k$. Hence, there exists some positive integer $N$ such that $f_{k}=0$ for every $k>N$. Thus, $g_{k}$ is an arbitrary function for any $k>N$, f can be represented as

$$
f(x)=\sum_{n=1}^{N}\left\langle f(x), e_{n}\right\rangle e_{n},
$$

and the expressions of the component functions $f_{n}$ and $g_{n}, \quad 1 \leq n \leq N$, of $\mathrm{f}$ and $\mathrm{g}$ come from statements (a) and (b) above. In the case where $H$ is finite- dimensional, the proof is clear.

As a consequence of Theorem 2.1 we derive formulas for the solutions of d'Alembert's Hilbert space valued functional equation (1.7).

Corollary 2.2. Let $M$ be a monoid, let $\sigma, \tau: M \rightarrow M$ be involutive automorphisms. Assume that the functions $g: M \rightarrow H$ satisfy (1.7). Then, there exists a positive integer $N$ such that

$$
f(x)=\sum_{n=1}^{N}\left\langle f(x), e_{n}\right\rangle e_{n} \text { and } x \rightarrow\left\langle g(x), e_{N+k}\right\rangle \text { is arbitrary }
$$

for all $x \in M$ and $k>0$. Furthermore, for every $k \in\{1,2, \ldots, N\}$, such that

$$
g(x)=\frac{1}{2} \sum_{k=1}^{N} \epsilon_{k}\left(\chi_{k}(x)+\chi_{k} \circ \sigma \circ \tau(x)\right) e_{k}, x \in M,
$$


where $\epsilon_{k}=1$ or 0 for every $k \in\{1,2, \ldots ., N\}$. for all $x \in M$, where $\chi_{k}$ is a non-zero multiplicative function of $M$ such that $\chi_{k} \circ \sigma \circ \tau=\chi_{k} \circ \tau \circ \sigma$

If $M$ is a topological monoid and $f \in C(M)$, then $\chi_{k}, \chi_{k} \circ \sigma \circ \tau \in C(M)$.

Proof. The proof follows by putting $f=g$ in Theorem 2.1.

Corollary 2.3. Let $M$ be a monoid, let $\tau: M \rightarrow M$ be involutive automorphisms. Assume that the functions $f, g: M \rightarrow H$ satisfy

$$
f(x y)+f(\tau(y) x)=2 g(x) * f(y) .
$$

Then, there exists a positive integer $N$ such that

$$
f(x)=\sum_{n=1}^{N}\left\langle f(x), e_{n}\right\rangle e_{n} \text { and } x \rightarrow\left\langle g(x), e_{N+k}\right\rangle \text { is arbitrary }
$$

for all $x \in M$ and $k>0$. Furthermore, for every $k \in\{1,2, \ldots, N\}$, we have the following possibilities:

$$
\left\{\begin{array}{l}
\left\langle g(x), e_{k}\right\rangle=\frac{\chi_{k}(x)+\chi_{k} \circ \tau(x)}{2} \\
\left\langle f(x), e_{k}\right\rangle=\frac{\alpha_{k}\left(\chi_{k}(x)+\chi_{k} \circ \tau(x)\right)}{2}
\end{array} ;\left\{\begin{array}{l}
\left\langle g(x), e_{k}\right\rangle \text { is an arbitrary function, } \\
\left\langle f(x), e_{k}\right\rangle=0
\end{array}\right.\right.
$$

for all $x \in M$, where $\chi_{k}$ is a non-zero multiplicative function of $M$ and $\alpha_{k} \in \mathbf{C} \backslash\{0\}$.

If $M$ is a topological monoid and $f \in C(M)$, then $\chi_{k}, \chi_{k} \circ \tau \in C(M)$.

Proof. The proof follows by putting $\sigma=i d$ in Theorem 2.1.

We complete this section with a result concerning Wilson Hilbert space valued functional equation (1.6).

Theorem 2.4. Let $M$ be a monoid which is generated by its squares, let $\sigma, \tau: M \rightarrow M$ be involutive automorphisms. Assume that the pair $f, g: M \rightarrow \mathbf{C}$, satisfy Wilson's Hilbert valued functional equation (1.6). Then, there exists a positive integer $N$ such that

$$
f(x)=\sum_{n=1}^{N}\left\langle f(x), e_{n}\right\rangle e_{n} \text { and }\left\langle g(x), e_{N+k}\right\rangle \text { is arbitrary }
$$

for all $x \in M$ and $k>0$. Furthermore, for every $k \in\{1,2, \ldots, N\}$, we have the following possibilities: 


$$
\left\{\begin{array}{l}
\left\langle g(x), e_{k}\right\rangle \text { is an arbitrary function, } \\
\left\langle f(x), e_{k}\right\rangle=0
\end{array} ;\left\{\begin{array}{l}
\left\langle g(x), e_{k}\right\rangle=\frac{\chi_{k}(x)+\chi_{k} \circ \sigma \circ \tau(x)}{2} \\
\left\langle f(x), e_{k}\right\rangle=\alpha_{k} \chi_{k} \circ \sigma(x)
\end{array}\right.\right.
$$

where $\chi_{k}: M \rightarrow \mathbf{C}$ is a non-zero multiplicative function with $\chi_{k} \circ \sigma \circ \tau=$ $\chi_{k} \circ \tau \circ \sigma$. and for some $\alpha_{k} \in \mathbf{C} \backslash\{0\}$.

(ii) There exists a non-zero multiplicative function $\chi_{k}: M \rightarrow \mathbf{C}$ with $\chi_{k} \circ \sigma \circ \tau=\chi_{k} \circ \tau \circ \sigma$ such that

$$
g_{k}=\frac{\chi_{k}+\chi_{k} \circ \sigma \circ \tau}{2} .
$$

Furthermore, we have

(1) If $\chi_{k} \neq \chi_{k} \circ \sigma \circ \tau$, then

$$
f_{k}=\alpha_{k} \chi_{k} \circ \sigma+\beta_{k} \chi_{k} \circ \tau
$$

for some $\alpha_{k}, \beta_{k} \in \mathbf{C} \backslash\{0\}$.

(2) If $\chi_{k}=\chi_{k} \circ \sigma \circ \tau$, then there exists a non-zero additive function $A_{k}: M \backslash I_{\chi_{k} \circ \sigma} \rightarrow \mathbf{C}$ with $A_{k} \circ \tau=-A_{k} \circ \sigma$ such that

$$
f_{k}(x)=\left\{\begin{array}{l}
\left(\alpha_{k}+A_{k}(x)\right) \chi_{k}(\sigma(x)) \text { for } x \in M \backslash I_{\chi_{k} \circ \sigma} \\
0 \text { for } x \in I_{\chi_{k} \circ \sigma}
\end{array}\right.
$$

for some $\alpha_{k}, \in \mathbf{C}$.

Conversely, if $f$ and $g$ have the forms described above, then the pair $(f, g)$ is a solution of equation (1.6). Moreover, if $M$ is a topological monoid generated by its squares, and $f, g \in C(M)$, then $\chi_{k}, \chi_{k} \circ \sigma, \chi_{k} \circ \tau, \chi_{k} \circ \sigma \circ \tau \in$ $C(M)$, while $A_{k} \in C\left(M \backslash I_{\chi_{k} \circ \sigma}\right)$.

Proof. We proceed as in the proof of Theorem 2.1. For every integer $k \geq 0$, we consider the functions $f_{k}, g_{k}: M \rightarrow \mathbf{C}$, defined by

$$
f_{k}(x)=\left\langle f(x), e_{k}\right\rangle \text { and } g_{k}(x)=\left\langle g(x), e_{k}\right\rangle \text { for } x \in M \text {. }
$$

Since the pair $(f, g)$ satisfies (1.6), for all $k \in \mathbf{N}$ we have

$$
f_{k}(x \sigma(y))+f_{k}(\tau(y) x)=2 f_{k}(x) g_{k}(y) \text { for all } x, y \in M \text {. }
$$

By [6,Theorem 3.4] we infer that there are only the following cases (a)

$$
f_{k}=0 \text { and } g_{k} \text { is an arbitrary function. }
$$


(b) There exists a non -zero multiplicative function $\chi_{k}: M \rightarrow \mathbf{C}$ such that

$$
f_{k}=\alpha_{k} \chi_{k} \circ \sigma \text { and } g_{k}=\frac{\chi_{k}+\chi_{k} \circ \sigma \circ \tau}{2}
$$

for some $\alpha_{k} \in \mathbf{C} \backslash\{0\}$.

(c) There exists a non-zero multiplicative function $\chi_{k}: M \rightarrow \mathbf{C}$ with $\chi_{k} \circ \sigma \circ \tau=\chi_{k} \circ \tau \circ \sigma$ such that

$$
g_{k}=\frac{\chi_{k}+\chi_{k} \circ \sigma \circ \tau}{2}
$$

Furthermore, we have.

(i) If $\chi_{k} \neq \chi_{k} \circ \sigma \circ \tau$, then

$$
f_{k}=\alpha_{k} \chi_{k} \circ \sigma+\beta_{k} \chi_{k} \circ \tau
$$

for some $\alpha_{k}, \beta_{k} \in \mathbf{C} \backslash\{0\}$.

(ii) If $\chi_{k}=\chi_{k} \circ \sigma \circ \tau$, then there exists a non-zero additive function $A_{k}: M \backslash I_{\chi_{k} \circ \sigma} \rightarrow \mathbf{C}$ with $A_{k} \circ \tau=-A_{k} \circ \sigma$ such that

$$
f_{k}(x)=\left\{\begin{array}{l}
\left(\alpha_{k}+A_{k}(x)\right) \chi_{k}(\sigma(x)) \text { for } x \in M \backslash I_{\chi_{k} \circ \sigma} \\
0 \text { for } x \in I_{\chi_{k} \circ \sigma}
\end{array}\right.
$$

for some $\alpha_{k} \in \mathbf{C}$. Conversely, the functions given with properties satisfy the functional equation (2.2). The continuation of the proof depends on the dimension of $H$. In fact, if $H$ is infinite-dimensional, then

$$
\left\langle g(x), e_{k}\right\rangle=g_{k}(x) \rightarrow 0 \text { as } k \rightarrow+\infty
$$

for every $x \in M$. Statements (b) and (c) are not possible for infinitely positive integers n. Hence, there exists some positive integer $N$ such that $f_{k}=0$ for every $k>N$. Thus, f can be represented as

$$
f(x)=\sum_{n=1}^{N}\left\langle f(x), e_{n}\right\rangle e_{n},
$$

$g_{k}$ is an arbitrary function for any $k>N$, and expressions of the component functions $f_{n}$ and $g_{n}, 1 \leq n \leq N$ of $f$ and $g$ follow from the previous discussion. In the case where $H$ is a finite-dimensional space, the proof is clear. 
Corollary 2.5. Let $M$ be a monoid which is generated by its squares, let $\tau: M \rightarrow M$ be an involutive automorphism, and let the pair $f, g: M \rightarrow H$ satisfy the functional equation

$$
f(x y)+f(\tau(y) x)=2 f(x) * g(y), \quad x, y \in M .
$$

Then, there exists a positive integer $N$ such that

$$
f(x)=\sum_{n=1}^{N}\left\langle f(x), e_{n}\right\rangle e_{n} \text { and } x \rightarrow\left\langle g(x), e_{N+k}\right\rangle \text { is arbitrary }
$$

for all $x \in M$ and $k>0$. Furthermore, for every $k \in\{1,2, \ldots, N\}$, we have the following possibilites:

(i) $\left\{\begin{array}{l}\left\langle g(x), e_{k}\right\rangle \text { is an arbitrary function, } \\ \left\langle f(x), e_{k}\right\rangle=0\end{array}\right.$

(ii) There exists a non-zero multiplicative function $\chi_{k}: M \rightarrow \mathbf{C}$ such that

$$
g_{k}=\frac{\chi_{k}+\chi_{k} \circ \tau}{2}
$$

Furthermore, we have.

(1) If $\chi_{k} \neq \chi_{k} \circ \tau$, then

$$
f_{k}=\alpha_{k} \chi_{k}+\beta_{k} \chi_{k} \circ \tau
$$

for some $\alpha_{k}, \beta_{k} \in \mathbf{C} \backslash\{0\}$.

(2) If $\chi_{k}=\chi_{k} \circ \tau$, then there exists an additive function $A_{k}: M \backslash I_{\chi_{k}} \rightarrow \mathbf{C}$ with $A_{k} \circ \tau=-A_{k}$ such that

$$
f_{k}(x)=\left\{\begin{array}{l}
\left(\alpha_{k}+A_{k}(x)\right) \chi_{k}(x) \text { for } x \in M \backslash I_{\chi_{k}} \\
0 \text { for } x \in I_{\chi_{k}}
\end{array}\right.
$$

for some $\alpha_{k} \in \mathbf{C}$.

Conversely, if $f$ and $g$ have the forms described above, then the pair $(f, g)$ is a solution. Moreover, if $M$ is a topological monoid generated by its squares, and $f, g \in C(M)$, then $\chi_{k}, \chi_{k} \circ \tau \in C(M)$, while $A_{k} \in C\left(M \backslash I_{\chi_{k}}\right)$.

Proof. The proof follows by putting $\sigma=i d$ in Theorem 2.4. 


\section{Superstability of Hilbert valued cosine type functional equa- tions}

The main result of this section is Theorem 3.3 that contains a superstability result for the functional equation (1.6). For the proof of our result we will begin by pointing out a superstability result for the equation

$$
f(x y)+f(\sigma(y) x)=2 f(x) g(y)
$$

where $f, g: G \rightarrow \mathbf{C}$ are the unknown functions.

Proposition 3.1. Let $\delta>0$ be given, let $M$ be a monoid and let $\sigma$ is an involutive morphism of $M$. Assume that the functions $f, g: M \rightarrow \mathbf{C}$ satisfies the inequality

$$
|f(x y)+f(\sigma(y) x)-2 f(x) g(y)| \leq \delta \text { for all } x, y \in M
$$

and that $g$ is unbounded. Then, the ordered pair $(f, g)$ satisfies equation (3.1).

Proof. The proof is part of the proof of [3,Theorem 2.1 and Theorem 3.7] if we put $\chi=1$ that deals with $M$ being a group.

Corollary 3.2. Let $\delta>0$ be given and let $G$ be a monoid. Assume that the function $f: G \rightarrow \mathbf{C}$ satisfies the inequality

$$
|f(x y)+f(\sigma(y) x)-2 f(x) f(y)| \leq \delta \text { for all } x, y \in G \text {. }
$$

Then, either

$$
|f(x)| \leq \frac{1+\sqrt{1+2 \delta}}{2} \text { for all } x \in G
$$

or $f$ has the form

$$
f=\frac{\mu+\mu \circ \sigma}{2},
$$

where $\mu$ is a multiplicative function.

Proof. The proof follows immediately from Propositon 3.1 and Theorem [1, Theorem 4]. 
Theorem 3.3. Let $\delta>0$ be given and let $M$ be a monoid. Assume that the functions $f, g: M \rightarrow H$ satisfy the inequality

$$
\|f(x y)+f(\sigma(y) x)-2 f(x) * g(y)\| \leq \delta \text { for all } x, y \in M
$$

Then, either

(i) there exists $k \geq 1$ such that the function $x \mapsto\left\langle g(x), e_{k}\right\rangle$ is bounded, or

(ii) the pair $(f, g)$ is a solution of the functional equation:

$$
f(x y)+f(\sigma(y) x)=2 f(x) * g(y) .
$$

Proof. Suppose that the pair $(f, g)$ satisfies (3.2). By applying the Parseval identity and the definition of Hadamard product with the inequality (3.2), we find that the scalar valued functions $f_{k}, g_{k}$ defined by

$$
f_{k}(x)=\left\langle f(x), e_{k}\right\rangle \text { and } g_{k}(x)=\left\langle g(x), e_{k}\right\rangle \text { for } x \in M,
$$

satisfy the inequality

$$
\left|f_{k}(x y)+f_{k}(\sigma(y) x)-2 f_{k}(x) g_{k}(y)\right| \leq \delta \text { for all } x, y \in M
$$

According to Proposition 3.1, for all $k \in \mathbf{N}$, we have that either the function $x \mapsto\left\langle g(x), e_{k}\right\rangle$ is bounded or the pair $\left(f_{k}, g_{k}\right)$ is a solution of (3.1). Then, we conclude that the pair $(f, g)$ satisfies equation (3.3) if assertion (i) fails.

In [4] it was proved that if $g: H \rightarrow H$ is surjective, then every component function $x \mapsto\left\langle g(x), e_{n}\right\rangle$ is unbounded. By applying Theorem (3.3), this leads to the following result.

Corollary 3.4. Let $\delta>0$ be given. Assume that functions $f, g: H \rightarrow H$, where $g$ is surjective, satisfy the inequality

$$
\|f(x y)+f(\sigma(y) x)-2 f(x) * g(y)\| \leq \delta \text { for all } x, y \in H .
$$

Then, the pair $(f, g)$ satisfies the equation

$$
f(x y)+f(\sigma(y) x)=2 f(x) * g(y) \text { for all } x, y \in H .
$$


Proof. Since g is surjective, then every component function $x \mapsto\left\langle g(x), e_{n}\right\rangle$ is unbounded. Thus, the proof follows immediately from Theorem 3.3.

Corollary 3.5. Let $\delta>0$ be given and let $G$ be a topological group. Assume that the function $g: G \rightarrow H$ satisfies the inequality

$$
\|g(x y)+g(\sigma(y) x)-2 g(x) * g(y)\| \leq \delta \text { for all } x, y \in G
$$

Then, either there exists $k \geq 1$ such that

$$
\left|\left\langle g(x), e_{k}\right\rangle\right| \leq \frac{1+\sqrt{1+2 \delta}}{2} \text { for all } x \in G
$$

or there exist a multiplicative function $\chi_{k}: M \rightarrow \mathbf{C} \backslash\{0\}$ and a positive integer $N$ such that

$$
g(x)=\frac{1}{2} \sum_{n=1}^{N} \epsilon_{n}\left(\chi_{k}(x)+\chi_{k} \circ \sigma(x)\right) e_{n}, \text { for all } x \in G,
$$

where $\epsilon_{n}=1$ or 0 for every $n \in\{1,2, \ldots ., N\}$.

Proof. If we put $f=g$ in Theorem 3.3, we immediately have that either there exists $k \geq 1$ such that the function $x \mapsto\left\langle g(x), e_{k}\right\rangle$ is bounded or $g$ is a solution of the equation

$$
g(x y)+g(\sigma(y) x)=2 g(x) * g(y), \quad x, y \in G .
$$

The remainder of the proof follows if we put $\chi=1$ from Corollary [3, Corollary 3.8] and Corollary 2.3.

Corollary 3.6. Let $\delta>0$ be given and let $G$ be a group with identity element. Let $g: G \rightarrow H$ such that

$$
\|g(x y)+g(y x)-2 g(x) * g(y)\| \leq \delta \text { for all } x, y \in G .
$$

Then either $g$ is bounded or $g$ is multiplicative.

Proof. From Corollary 2.2 and Corollary 2.5 and then using [3, Corollary $3.9]$. 


\section{References}

[1] J. A. Baker, The stability of the Cosine Equation, Proc. Amer. Math. Soc. 80, 3, pp. 411-416, (1980).

[2] A. Chahbi, B. Fadli, S. Kabbaj, A generalization of the symmetrized multiplicative Cauchy equation. Acta Math. Hung. 149 (1), pp. 170176, (2016).

[3] E. Elqorachi and A. Redouani, Solutions and stability of variant of Wilson's functional equation (14 May 2015), arXiv:1505.06512v1 [math. $\mathrm{CA}$. Demonstratio Math. (to appear).

[4] H. Rezaei and M. Sharifzadeh, On the super-stability of exponential hilbert valued functional equations, J. Inequal. Appl. Article ID 114. (2011).

[5] L. Székelyhidi, D'Alembert's functional equation on compact groups, Banach J.. Math. Anal. 1, no. 2, pp. 221-226, (2007).

[6] KH. Sabour, B. Fadli and S. Kabbaj, Wilson's Functional equation on monoids with involutive automorphisms. Aequationes. Math. 90, no. 5, pp. 189-196, (2016).

[7] H. Stetkaer, D'Alembert's and Wilson's functional equations on step 2 nilpotent groups, Aequationes. Math. 67, No. 3, pp. 1001-1011, (2004).

[8] H. Stetkaer, Functional Equations on Groups, World Scientific Publishing Co., Singapore, (2013).

[9] H. Stetkaer, A link between Wilson's functional and d'Alembert's functional equations, Aequationes. Math. 90, No. 2, pp. 407-409, (2016).

[10] W. H. Wilson, On a certain related functional equations, Proc. Amer. Math. Soc. 26, pp. 300-312, (1920).

[11] D. Zeglami, M. Tial and B. Fadli, Wilson's type Hilbert space valued functional equations. Adv. Pure Appl. Math. 7 (3), pp. 189-196, (2016). 


\section{Hajira Dimou}

Department of Mathematics,

Faculty of Sciences

Ibn Tofail University, Kenitra,

Morocco

e-mail : dimouhajira@gmail.com

and

\section{Samir Kabbaj}

Department of Mathematics,

Faculty of Sciences

Ibn Tofail University,

Kenitra,

Morocco

e-mail : samkabbaj@yahoo.fr 